\title{
Review
}

\section{Cell adhesion molecules nectins and associating proteins: Implications for physiology and pathology}

\author{
By Hisakazu OGIta,${ }^{* 1}$ Yoshiyuki RIKITAKe, ${ }^{* 1, * 2}$ Jun MiYOSHI ${ }^{* 3}$ and Yoshimi TAKAI ${ }^{* 1, \dagger}$ \\ (Communicated by Shigetada NAKANISHI, M.J.A.)
}

\begin{abstract}
Nectins have recently been identified as new cell adhesion molecules (CAMs) consisting of four members. They show immunoglobulin-like structures and exclusively localize at adherens junctions (AJs) between two neighboring cells. During the formation of cell-cell junctions, nectins function in cooperation with or independently of cadherins, major CAMs at AJs. Similar to cadherins, which are linked to the actin cytoskeleton by binding to catenins, nectins also bind to afadin through their C-terminal region and are linked to the actin cytoskeleton. In addition to nectins, there are nectin-like molecules (Necls), which resemble nectins in their structures and consist of five members. Nectins and Necls are involved in the formation of various kinds of cell-cell adhesion, and also play key roles in diverse cellular functions including cell movement, proliferation, survival, and differentiation. Thus, nectins and Necls are crucial for physiology and pathology of multicellular organisms.
\end{abstract}

Keywords: afadin, integrin, nectin, nectin-like molecule, receptor

\section{Introduction}

Cell adhesion includes cell-cell and cell-matrix junctions at which various cell adhesion molecules (CAMs) and their interacting proteins are identified and characterized. Cell-cell junctions are mainly classified into three categories: 1) symmetric junctions between identical cell types, such as epithelial cells and endothelial cells, 2) asymmetric junctions, which are usually observed at synapses that are formed between the axon of one neuron and the

*1 Division of Molecular and Cellular Biology, Department of Biochemistry and Molecular Biology, Kobe University Graduate School of Medicine, Hyogo, Japan.

*2 Division of Signal Transduction, Department of Biochemistry and Molecular Biology, Kobe University Graduate School of Medicine, Hyogo, Japan.

*3 Department of Molecular Biology, Osaka Medical Center for Cancer and Cardiovascular Diseases, Osaka, Japan.

$\dagger$ Correspondence should be addressed: Y. Takai, Division of Molecular and Cellular Biology, Department of Biochemistry and Molecular Biology, Kobe University Graduate School of Medicine, 7-5-1 Kusunoki-cho, Chuo-ku, Kobe, Hyogo 650-0017, Japan (e-mail: ytakai@med.kobe-u.ac.jp).

Abbreviations: CAM: cell adhesion molecule; AJ: adherens junction; TJ: tight junction; Necl: nectin-like molecule; JAM: junctional adhesion molecule; PDGF: platelet-derived growth factor. dendrite of another neuron, and 3) heterotypic intercellular junctions, which include those between differentiating germ cells and their supporter Sertoli cells in the testis and between specialized sensory cells and supporting cells in the sensory epithelia. These kinds of cell-cell junctions play essential roles in the formation and maintenance of tissues and organs in multicellular organisms. Cell-cell junctions comprise several specialized junctional apparatuses including adherens junctions (AJs), tight junctions (TJs), desmosomes, and gap junctions. AJs serve as a mechanically adhesive apparatus between neighboring cells. TJs are localized at the most apical side of cell-cell junctions in epithelial cells. Two major functions of TJs are to act as a barrier, preventing the passage of soluble molecules through the gaps between cells, and as a fence, keeping the cell surface proteins and lipids in the basolateral region separate from those in the apical region. ${ }^{1), 2)}$ Similar to AJs, desmosomes physically connect neighboring cells and are also necessary for the stability of AJs.3) Gap junctions contain channels between two cells through which the direct intercellular transmission of molecules is enabled. ${ }^{4)}$ At cell-matrix junctions, there are also some specialized apparatuses: focal complexes and focal adhesions. Focal complexes are small 


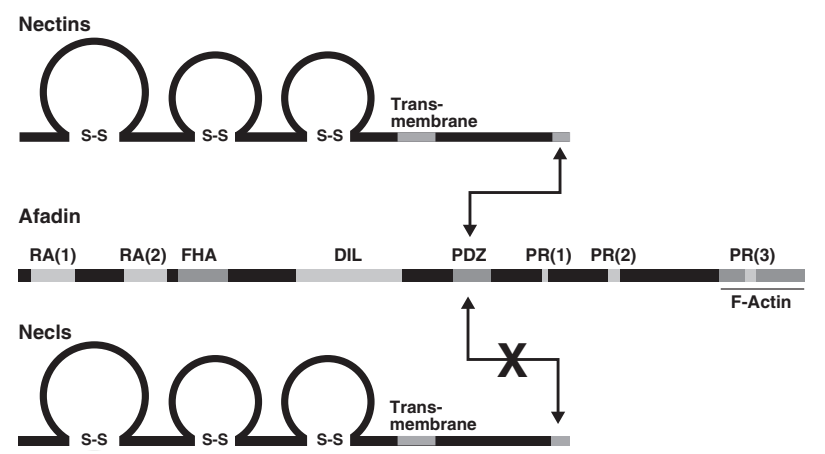

Fig. 1. Molecular structures of nectins, Necls and afadin. Nectins and Necls contain three immunoglobulin-like loops in their extracellular region, a single transmembrane segment and a cytoplasmic tail. The nectin family members possess a consensus motif of C-terminal four amino acids for interaction with afadin, which in turn interacts with F-actin to connect nectins to F-actin. Direct binding between nectins and afadin is conducted through the C-terminal motif of nectins and the PDZ domain of afadin. Necls are structurally similar to nectins, but do not directly bind to afadin. DIL, dilute domain; FHA, forkhead-associated domain; PDZ, PDZ domain; RA, Rasassociation domain; PR, proline-rich domain.

$(<0.5 \mu \mathrm{m}$ diameter $)$ immature cell-matrix junctions that are observed at the peripheral region of the leading edge of moving cells, and focal adhesions are formed just at the rear side of focal complexes to firmly anchor cells to extracellular matrix. The dynamic regulation of formation and disruption of focal complexes and focal adhesions is crucial for effective cell movement.

We have indentified a new cell adhesion system consisting of nectins and afadin at AJs. ${ }^{5}{ }^{5}$ Nectins are immunoglobulin-like CAMs and the nectin family comprises four members (nectin-1 through nectin-4), while a single afadin gene was identified to date (Fig. 1). Afadin binds to nectins at their C-terminal region and connects them to the actin cytoskeleton. Nectins and afadin play roles in the formation of various cell-cell junctions cooperatively with or independently of cadherins, major CAMs at AJs. In addition to the role of cell-cell junction formation, nectins regulate multiple cellular functions, such as cell polarity, movement, proliferation, differentiation, and survival, in cooperation with integrins, growth factor receptors, and nectin-like molecules (Necls). Necls resemble nectins in their molecular structure, but do not bind to afadin (Fig. 1). The Necl family comprises five members (Necl-1 through Necl-5). Our recent studies on nectins and Necls could contribute to the understanding of pathology of many diseases. In this review article, physiological and pathological roles of cell adhesions are described by focusing on new CAMs nectins and Necls. General properties and functions of nectins and Necls have been described in our previous reviews in detail. ${ }^{6)-12)}$

\section{Properties of CAMS at AJs}

i. Cadherins and catenins. Cadherins have been known as the main components of AJs. ${ }^{13)}$ The extracellular domain of cadherins on the surface of one cell binds to that on the surface of another cell in a $\mathrm{Ca}^{2+}$-dependent manner (this engagement is called "homophilic interactions in trans"), leading to the formation of apposing cell adhesion. On the other hand, the intracellular domain of cadherins binds to $\beta$-catenin, which in turn binds to an F-actin-binding protein $\alpha$-catenin. AJs are formed by the assembly of multiple interactions of cadherins in trans and the intracellular linkage of the cadherin-catenin system to the actin cytoskeleton.

ii. Nectins and afadin. Nectins and afadin have been identified in our laboratory and their roles in cell adhesion have been vigorously investigated. ${ }^{6)}{ }^{-12)}$ It is clear that nectins firstly form rather weak cell adhesion and then recruit cadherins to the nectin-mediated cell-cell contacts to establish AJs. Similar to cadherins, nectins interact in trans with each other through their extracellular domains to make contacts between the opposing membranes of the cells. Nectin engagement is $\mathrm{Ca}^{2+}$-independent, whereas cadherin engagement is $\mathrm{Ca}^{2+}$-dependent as described above. The intracellular domain of nectins binds to an F-actin-binding protein afadin, linking nectins to the actin cytoskeleton. Besides nectins, afadin can directly bind to $\alpha$-catenin and other related proteins. Thus, the afadin-mediated assembly of multiple components, including the cadherin- $\beta$ catenin complex, at the nectin-initiated cell-cell contact sites contributes to the establishment of AJs.

\section{Molecular mechanisms of the formation of cell-cell junctions}

When growth factors or neurotransmitters bind to their cognate receptors, the receptors change their conformation and the intracellular signaling molecules are activated, eventually leading to the induction of various cellular functions. Similarly, the trans-interaction of nectins induces the activation of the intracellular signaling (Fig. 2). c-Src is first activated after the trans-interaction of nectins. ${ }^{14)}$ Activated c-Src then activates Rap1, Cdc42, and Rac small G proteins. Rap1 activated in this way subsequently binds to afadin, and Cdc42 and Rac also binds to their downstream effectors such as 


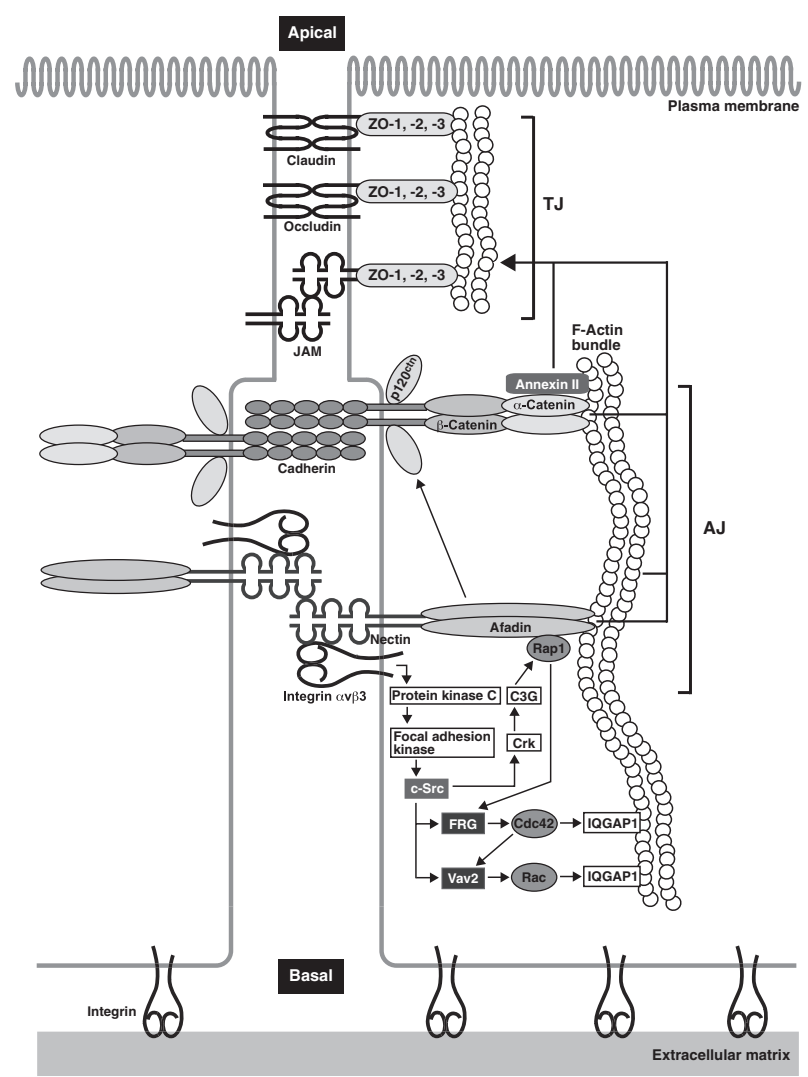

Fig. 2. Nectin-induced formation of AJs and TJs. Trans-interacting nectin at the initial cell-cell adhesion induces the activation of Rap1, Cdc42, and Rac mediated by c-Src, Crk, C3G, FRG, and Vav2. This nectin-induced signaling is dependent on integrin $\alpha \mathrm{v} \beta 3$, which physically associates with nectin, and its downstream signaling molecules protein kinase $\mathrm{C}$ and foca adhesion kinase. Activated $\mathrm{Cdc} 42$ and Rac reorganize the actin cytoskeleton and recruit the cadherin-catenin system to the nectin-based cell-cell adhesion sites. At this phase, cadherin has only weak adhesion activity. However, afadin interacting with activated Rap1 also associates with p120 ${ }^{\mathrm{ctn}}$, leading to increased adhesion activity of cadherin and the establishment of cadherinbased AJs. After the formation of AJs, both the nectin-afadin and cadherin-catenin systems cooperatively play an essential role in the formation of TJs as well as AJs. The reorganized actin cytoskeleton and actin binding proteins including annexin II and IQGAP1 are also involved in the formation of TJs.

IQGAP1, IRSp53/WAVE, NWASP, and WASP. Since these binding proteins are all capable of interacting with F-actin, nectins can regulate reorganization of the actin cytoskeleton at the nectinbased cell-cell adhesion sites through these proteins. $\alpha$-Catenin also binds to the reorganized actin cytoskeleton and then recruits the cadherin- $\beta$-catenin complex. Therefore, both the direct interaction of afadin with $\alpha$-catenin (details are described in the above section) and the reorganization of the actin cytoskeleton cooperatively contribute to the recruitment of the cadherin-catenin complex to the nectinbased cell-cell adhesion sites, eventually resulting in the establishment of AJs.

Integrins play a major role in the formation of cell-matrix junctions. Our recent study reveals that integrin $\alpha \mathrm{v} \beta 3$ physically interacts in cis with nectins and is necessary for the nectin-induced intracellular signaling (Fig. 2). Activated integrin $\alpha \mathrm{v} \beta 3$, which shows high-affinity for its substrate vitronectin, sequentially activates protein kinase $\mathrm{C}$ and focal adhesion kinase. ${ }^{15)}$ Since the activation of these kinases results in the activation of c-Src, this cSrc activation downstream of the trans-interaction of nectins as described above is actually induced by cooperative roles of nectins and integrin $\alpha \mathrm{v} \beta 3$. Based on these results, integrin $\alpha \mathrm{v} \beta 3$ is involved in the formation of cell--cell junctions as well as cell-matrix junctions.

In epithelial cells, TJs are always formed at the apical side of AJs due to cell polarization along the apical-basal axis on the interface between two cells. Nectin-induced intracellular signaling, the reorganized actin cytoskeleton, and afadin are also important for the formation of TJs as well as AJs (Fig. 2). After or during the formation of AJs, nectin recruits first immunoglobulin-like CAMs junctional adhesion molecules (JAMs) and then other CAMs claudins and occludin to the apical side of AJs in cooperation with afadin, leading to the formation of $\mathrm{TJS}^{5}{ }^{5}$ $\mathrm{ZO}$ proteins, which are F-actin-binding proteins, interact with the cytoplasmic region of TJ constituents JAMs, occludin, and claudins. These CAMs are linked to the actin cytoskeleton through $\mathrm{ZO}$ proteins. Prior to the formation of T.Js, ZO-1, a member of $\mathrm{ZO}$ proteins, transiently interacts with afadin. This interaction is additionally necessary for the localization of JAM and claudin at the apical side of AJs to form TJs. However, the entire molecular mechanisms of the recruitment of the TJ constituents at the apical side of AJs remains largely unclear.

\section{Nectin-dependent and cadherin-independent cell adhesion}

Although cadherins are involved in many types of cell-cell adhesion in the multicellular organisms, it is indicated that there exist cell adhesions that are not mediated by cadherins, but solely by nectins. For instance, heterotypic interactions between nectin- 2 and nectin-3 play important roles during spermatogenesis (Fig. 3A). ${ }^{16)}$ To the full development of germ cells, progenitor cells form weak and easily removable 
A

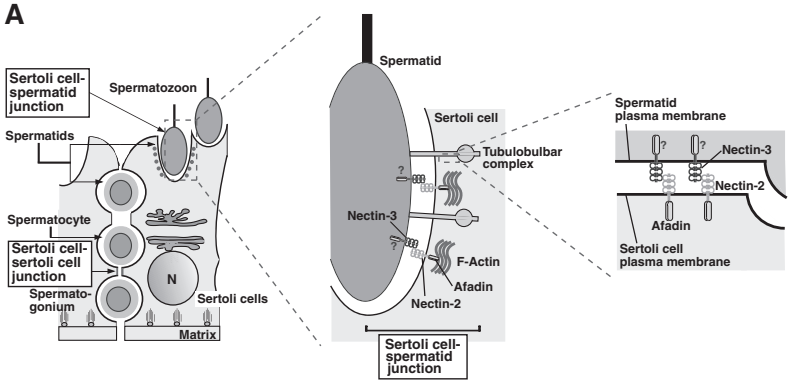

B

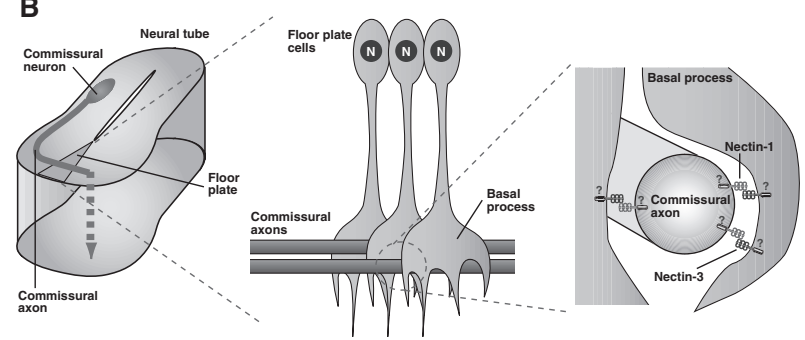

C

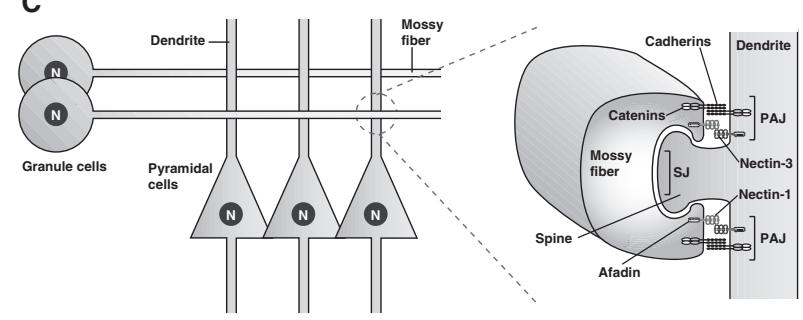

Fig. 3. Nectin-dependent and cadherin-independent cell adhesion. (A) Sertoli cell-spermatid junctions in the testis. (B) Contacts between the commissural axons and the floor plate cells in the neural tube. (C) Synapses between the mossy fiber terminals and dendrites of pyramidal cells in the CA3 region of the hippocampus. Question marks in the figures indicate that the existence of afadin and/or the interaction of nectins with afadin are not definitely confirmed.

contacts with Sertoli cells during differentiation from spermatogonia to sperm cells in the testes. The asymmetric distribution of nectin- 2 in Sertoli cells and nectin-3 in germ cells confers an advantage on selective adhesion between these cells. Moreover, nectins, but not cadherins, play roles in cell adhesion between the extending axons and dendrites of the floor plate cells in the spinal cord (Fig. 3B). ${ }^{17)}$ When the commissural axons make cell contacts with the dendrites of the floor plate cells, they extend across the central canal to make shift either to the rostral side or to the caudal side. The asymmetric distribution of nectin-3 in extending axons and nectin- 1 in dendrites of the floor plate cells confers a selective binding advantage for these cells. Thus, heterotypic interactions of nectins are supposed to be useful for transient cell contacts implicated in exchanging intercellular signals or for cell contacts subsequently followed by gross morphological changes that depend on actin dynamics.

\section{Neuronal synapses}

Neuronal cells generate one axon and numerous dendrites. An axon makes contact with dendrites to form synapses. Synapses are necessary for memory formation and preservation. Furthermore, neuronal activation induces the synaptic remodeling, which participates in long-term cortical plasticity. Synapse formation has previously been explained by the functions of cadherins. However, our studies have made it clear that nectins mediate the initial step of contacts between axons and dendrites and then recruit cadherins to form mature synaptic junctions. On the other hand, it has been unanswered why an axon makes contacts with dendrites, but not with other axons, and why an dendrite makes contacts with axons, but not with other dendrites. In collaboration with Dr. Takeichi's group, we found that the asymmetric localization of nectin- 1 at the terminus of an axon and nectin- 3 at dendrites plays major roles in defining selective interactions between an axon and dendrites (Fig. 3C). ${ }^{18)}$ Moreover, the nectin-afadin complex takes part in the assembly of molecules, such as Bassoon, Piccolo, CAST, and RIM1, at the active zone of the pre-synapse and in the assembly of molecules, such as PSD-95 and SSCAM, at the post-synaptic density. Afadin is also known to play roles in synaptic remodeling and the formation in the mouse hippocampus. ${ }^{19)}$

\section{Cell movement}

i. Enhancement of cell movement. When fibroblasts and endothelial cells move towards higher concentrations of platelet-derived growth factor (PDGF) and vascular endothelial growth factor, respectively, they need to dynamically form protrusions such as lamellipodia and filopodia at the leading edge and peripheral ruffles over the lamellipodia. ${ }^{20), 21)}$ In fibroblasts, the PDGF-induced activation of PDGF receptor and its downstream signaling as well as the vitronectin-mediated activation of integrin $\alpha \mathrm{v} \beta 3$ and its downstream signaling are important for the formation of these leading edge structures. ${ }^{22)}$ In particular, Rho, Rac, and Cdc42 small G proteins downstream of PDGF receptor and integrin $\alpha \mathrm{v} \beta 3$ play crucial roles in the formation of these leading edge structures. However, we demonstrated that the actions of PDGF receptor and integrin $\alpha \mathrm{v} \beta 3$ are insufficient for the full activation of these signaling 
A Leading edge of moving and proliferating cell

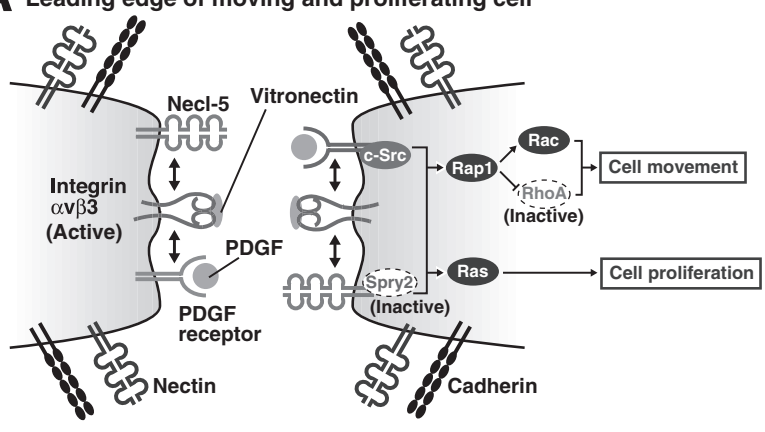

B

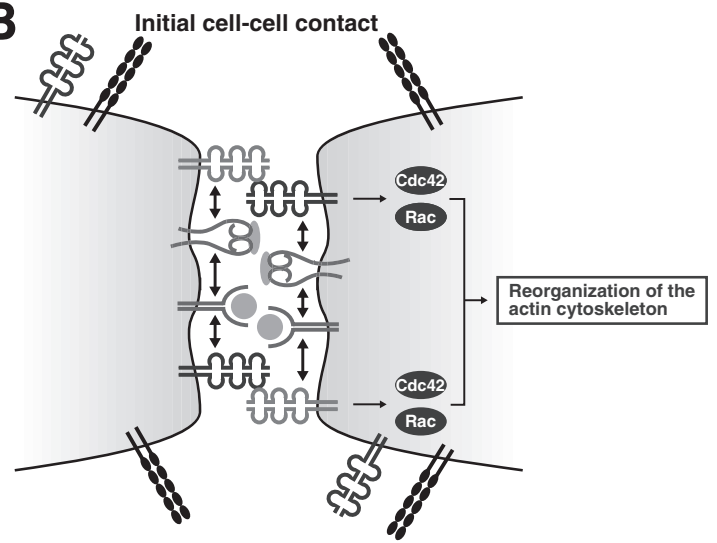

C Formation of nectin-based cell-cell adhesion
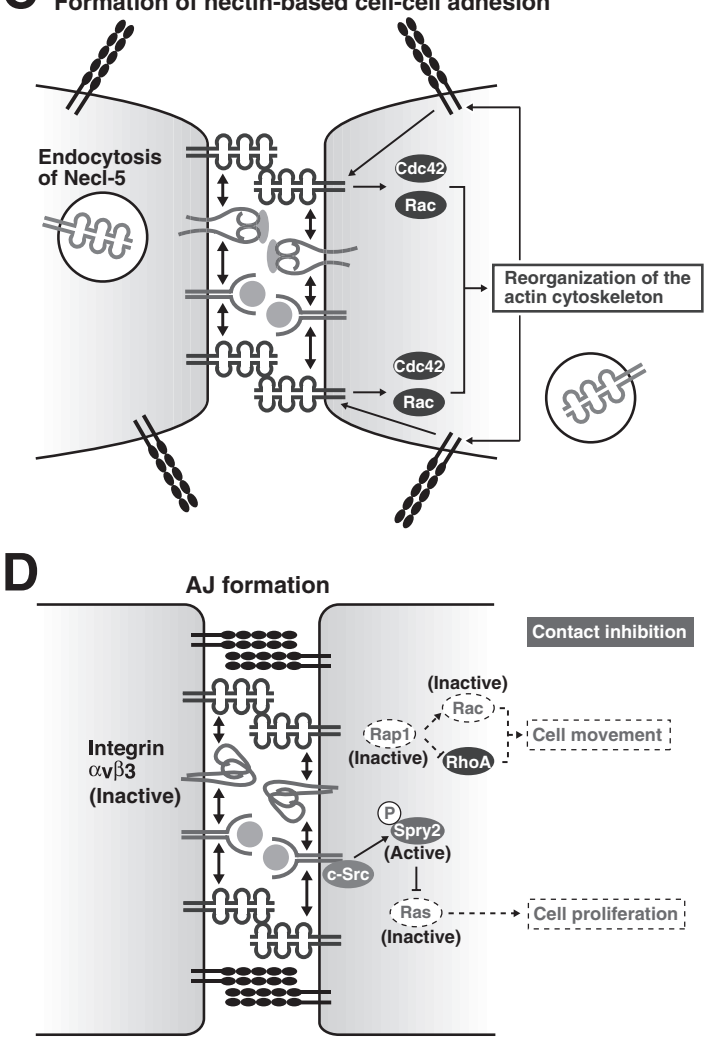

molecules, and that Necl-5 is additionally required for this full activation by forming a complex with PDGF receptor and integrin $\alpha \mathrm{v} \beta 3 .{ }^{23), 24)}$ The Necl-5-PDGF receptor-integrin $\alpha \mathrm{v} \beta 3$ complex fully activates intracellular signaling molecules, including Rac, upon PDGF stimulation. The activation of Rac induces the formation of lamellipodia and filopodia at the leading edge, and the reorganization of the actin cytoskeleton, which in turn promotes the accumulation of PDGF receptor, integrin $\alpha \mathrm{v} \beta 3$, and Necl-5 at the leading edge. As a result of the positive feedback mechanism, the formation of the leading edge occurs rapidly, resulting in the enhancement of cell movement in the direction of higher concentration of PDGF.

ii. Dynamics during cell movement. Leading edge structures are dynamically assembled and disassembled during cell movement. When PDGF binds to PDGF receptor, Rap1 is activated by a mechanism dependent on Necl-5 and integrin $\alpha \mathrm{v} \beta 3 .^{25)}$ Activated Rap1 induces the activation of Rac and the inactivation of RhoA (Fig. 4A). ${ }^{25), 26)}$ The activation of Rap1 is dynamically regulated by afadin that does not interact with nectins and by a Rap GAP, SPA-1. The inactivation of RhoA is dynamically regulated by a Rap-dependent Rho GAP, ARAP1. The cyclical activation and inactivation of these small $\mathrm{G}$ proteins essentially regulate the formation of leading edge structures.

Fig. 4. Regulation of cell movement and proliferation by nectins and Necl-5. (A) Necl-5, integrin $\alpha \mathrm{v} \beta 3$ and PDGF receptor form a complex at the leading edge of a moving cell and enhance cell movement by inducing c-Src and Rap1 activation, which leads to the activation of Rac together with the inhibition of RhoA. The ternary complex also enhances cell proliferation by inducing the activation of the Ras-mediated signalling pathway through inhibiting Necl-5-associated sprouty2 (Spry2). At this stage, nectins and cadherins are sparsely distributed on the cell surface. (B) The initial cell-cell contact is formed by the transinteraction of nectin-3 with Necl-5. At this stage, the reorganization of the actin cytoskeleton starts with the activation of Cdc42 and Rac. (C) The trans-interaction of nectin-3 with Necl5 is transient, and Necl-5 is subsequently downregulated from the cell surface by endocytosis. Next, trans-interaction of nectins occurs. (D) Cadherins are recruited to nectin-based cell-cell adhesion sites and homophilically interact in trans to form adherens junctions (AJs). At this stage, integrin $\alpha \mathrm{v} \beta 3$ becomes inactive by the action of trans-interacting nectins. Due to the downregulation of Necl-5 from the cell surface, Spry2 is released from Necl-5 and is tyrosine-phosphorylated by c-Src and becomes active to inhibit the Ras-mediated cell proliferation signals. The intracellular signalling mediated by integrin $\alpha \mathrm{v} \beta 3$ and PDGF receptor is then suppressed, resulting in the inhibition of cell movement and proliferation (contact inhibition). 
iii. Directionality of cell movement. Cells move in the direction of higher concentrations of growth factors and chemokines. However, mechanisms that regulate the directionality of cell movement are poorly understood. We revealed that afadin, which localizes at the leading edge, does not interact with nectins and plays an important role in the determination of the directionality. ${ }^{27)}$ Afadin facilitates the stabilization of the formation of leading edge structures in association with the activation of small G proteins, such as Rap1 and Rac. Although leading edge structures are formed in the direction of higher concentration of chemoattractants, the initial formation of the leading edge occurs incidentally. When a receptor at the initial leading edge is consecutively stimulated by a chemoattractant, the accumulation of receptors at the leading edge is induced by the activation of Rac and the reorganization of the actin cytoskeleton in a positive feedback manner, as described above. Since afadin regulates this activation of Rac and the reorganization of the actin cytoskeleton, it plays an indispensable role in the accumulation of the receptor at the leading edge.

\section{Contact inhibition of cell movement and proliferation}

Normal cells proliferate while individually moving. Cell movement and proliferation stop when the cell comes in contact with each other. This phenomenon is referred to as contact inhibition of cell movement and proliferation, which has been known for more than 50 years, ${ }^{28), 29)}$ and is extremely important for the tissue and organ generation during the development and for the wound healing process. Contact inhibition disappears when the cells are transformed, leading to the abnormal proliferation and the acquisition of invasive and metastatic potentials in transformed cells. ${ }^{30), 31)}$ We reported the novel mechanism that regulates contact inhibition of cell movement and proliferation, ${ }^{32)}$ although many possible mechanisms of contact inhibition have been proposed so far. When cells do not make contacts with and are free from other cells, these cells move and proliferate. In these cells, Necl-5 forms the ternary complex with PDGF receptor and integrin $\alpha \mathrm{v} \beta 3$ at the leading edge and increases cell movement by the mechanism as described in the above section and cell proliferation by enhancing the activation of the signaling pathway that includes Ras, Raf, MEK, and ERK (Fig. 4A). ${ }^{33)}$ When the moving and proliferating cells contact with each other, Necl-5 that locates at the leading edge first interacts with nectin-3
(Fig. 4B). This interaction triggers the internalization of Necl-5 from the cell surface by the clathrindependent endocytosis (Fig. 4C). Then, PDGF receptor and integrin $\alpha \mathrm{v} \beta 3$ are released from Necl-5 and interact with nectins. Nectins form cell-cell adhesion by the mechanism as mentioned above, which results in the termination of cell movement and proliferation (Fig. 4D). Moreover, after cell-cell adhesion is formed, nectins inactivate integrin $\alpha \mathrm{v} \beta 3$, which also contributes to the inhibition of cell movement to maintain cell-cell adhesion. ${ }^{34)}$ In addition, Necl-5 stops cell proliferation by regulating Sprouty2, a negative regulator for the Ras-mediated signaling. ${ }^{35)}$ The down-regulation of Necl-5 causes the tyrosine phosphorylation of Sprouty2 by c-Src, which is activated by $\mathrm{PDGF}$ receptor in response to PDGF, and inhibits the PDGF-induced Ras signaling.

\section{Implications of nectins and Necls for pathology of diseases}

Since Necl-5 and integrin $\alpha \mathrm{v} \beta 3$ are overexpressed in cancer cells, the down-regulation of Necl5 is perturbed in cancer cells even after making contacts with each other, which cancels contact inhibition of cell movement and proliferation and causes the abnormal proliferation, invasion, and metastasis. Necl-2 was identified as a tumor suppressor and its expression disappears in cancer cells. We have recently elucidated the mechanism of Necl-2 in tumor suppression. ${ }^{36)}$ ErbB2 and ErbB3, epidermal growth factor receptor family members, form a heterodimer. When the ligand binds to this heterodimer, Rac and Akt are activated, followed by the increase in cell movement and the suppression of cell death, respectively. Necl-2, which is expressed in normal epithelial cells, interacts in cis with ErbB3 through their extracellular regions, and binds to a tyrosine-phosphatase PTPN13 through its intracellular region. In normal epithelial cells, PTPN13 recruited to Necl-2 dephosphorylates the ErbB2mediated phosphorylation of ErbB3, impairing the activation of Rac and Akt. On the other hand, in cancer cells, these signalings are activated due to the disappearance of Necl-2, resulting in the promotion of invasion and metastasis.

Nectins and Necl-5 are also entry receptors for herpes virus and poliovirus, respectively, and are involved in the invasion of these viruses into host cells. In addition, mutations in human nectin-1 are also responsible for cleft lip/palate-ectodermal dysplasia, which includes Zlotogora-Ogur syndrome and Margarita Island ectodermal dysplasia and is an 
autosomal recessive disorder, clinically characterized by unusual faces, dental anomalies, hypotrichosis, palmoplantar hyperkeratosis and onychodysplasia, syndactyly, cleft lip/palate, and in some cases, mental retardation. ${ }^{37)}$

\section{Concluding remarks}

A series of our studies have clearly established that nectins and cadherins play key roles in the cellcell adhesion. Nectins regulate a variety of important cellular functions in addition to the cell-cell adhesion in cooperation with a lot of other molecules such as afadin, Necls, integrins, and cell surface receptors. Some properties of the adhesive function are different between nectins and cadherins. Nectins, but not cadherins, initiate cell-cell adhesion between two opposing cells, because the trans-interaction of nectins occurs earlier than that of cadherins. Another distinct feature is that cadherins only trans-interact homophilically with each other. Namely, E-cadherin expressed in epithelial cells interacts with E-cadherin, but not N-cadherin expressed in fibroblasts and neurons. In contrast, nectins homophilically and heterophilically trans-interact with each other. This difference is important for the physiologic function of these two CAMs. Cadherins play an important role in the tissue generation composed of homologous cells in which homophilic cell-cell junctions are formed. However, since various kinds of cells exist in most tissues and organs, cell-cell junctions are formed between heterogenic cells. Nectins, which are able to homophilically and heterophilically trans-interact with each other, play a critical role in the formation of these heterotypic intercellular junctions. We mainly depict the functions of nectins, Necls, and afadin in terms of cell adhesion, movement, proliferation, and synapse formation. In addition to such cellular functions, these molecules are also involved in the regulation of cell polarization, survival, and differentiation. ${ }^{38)-40)}$ Based on the diverse properties of nectins, Necls, and afadin, these molecules are likely to be more important than cadherins and catenins for such physiological and pathological processes. This is one of the research topics in the field of cell adhesion and thus should be vigorously clarified in the future.

\section{Acknowledgements}

Former faculty members, Drs. H. Nakanishi (Kumamoto University, Kumamoto, Japan), K. Irie (University of Tsukuba, Ibaraki, Japan), K. Shimizu (Kobe Medical Center, Kobe, Japan), T. Sakisaka
(Kobe University, Kobe, Japan), and W. Ikeda (Kan Research Institute, Kobe, Japan), many graduate students, post-doctoral fellows, and collaborators made great contributions to this work. We thank them for their excellent achievement. This work was supported by Grants-in-Aid for Scientific Research and for Cancer Research from the Ministry of Education, Culture, Sports, Science and Technology, Japan (2009-2010).

\section{References}

1) Tsukita, S., Furuse, M. and Itoh, M. (1999) Structural and signalling molecules come together at tight junctions. Curr. Opin. Cell Biol. 11, 628 633.

2) Tsukita, S. and Furuse, M. (2002) Claudin-based barrier in simple and stratified cellular sheets. Curr. Opin. Cell Biol. 14, 531-536.

3) Garrod, D.R., Merritt, A.J. and Nie, Z. (2002) Desmosomal cadherins. Curr. Opin. Cell Biol. 14, 537-545.

4) Kumar, N.M. and Gilula, N.B. (1996) The gap junction communication channel. Cell 84, 381-388.

5) Takai, Y. and Nakanishi, H. (2003) Nectin and afadin: novel organizers of intercellular junctions. J. Cell Sci. 116, 17-27.

6) Miyoshi, J. and Takai, Y. (2008) Structural and functional associations of apical junctions with cytoskeleton. Biochim. Biophys. Acta 1778, 670691.

7) Nakanishi, H. and Takai, Y. (2008) Frabin and other related Cdc42-specific guanine nucleotide exchange factors couple the actin cytoskeleton with the plasma membrane. J. Cell. Mol. Med. 12, 11691176 .

8) Ogita, H. and Takai, Y. (2008) Cross-talk among integrin, cadherin, and growth factor receptor: roles of nectin and nectin-like molecule. Int. Rev. Cytol. 265, 1-54.

9) Rikitake, Y. and Takai, Y. (2008) Interactions of the cell adhesion molecule nectin with transmembrane and peripheral membrane proteins for pleiotropic functions. Cell. Mol. Life Sci. 65, 253-263.

10) Sakisaka, T., Ikeda, W., Ogita, H., Fujita, N. and Takai, Y. (2007) The roles of nectins in cell adhesions: cooperation with other cell adhesion molecules and growth factor receptors. Curr. Opin. Cell Biol. 19, 593-602.

11) Takai, Y., Ikeda, W., Ogita, H. and Rikitake, Y. (2008) The Immunoglobulin-like Cell Adhesion Molecule Nectin and Its Associated Protein Afadin. Annu. Rev. Cell Dev. Biol. 24, 309-342.

12) Takai, Y., Miyoshi, J., Ikeda, W. and Ogita, H. (2008) Nectins and nectin-like molecules: roles in contact inhibition of cell movement and proliferation. Nat. Rev. Mol. Cell Biol. 9, 603-615.

13) Takeichi, M. (1988) The cadherins: cell-cell adhesion molecules controlling animal morphogenesis. Development 102, 639-655. 
14) Fukuhara, T., Shimizu, K., Kawakatsu, T. Fukuyama, T., Minami, Y., Honda, T. et al. (2004) Activation of Cdc42 by trans interactions of the cell adhesion molecules nectins through c-Src and Cdc42-GEF FRG. J. Cell Biol. 166, 393-405.

15) Ozaki, M., Ogita, H. and Takai, Y. (2007) Involvement of integrin-induced activation of protein kinase $\mathrm{C}$ in the formation of adherens junctions. Genes Cells 12, 651-662.

16) Inagaki, M., Irie, K., Ishizaki, H., Tanaka-Okamoto, M., Miyoshi, J. and Takai, Y. (2006) Role of cell adhesion molecule nectin-3 in spermatid development. Genes Cells 11, 1125-1132.

17) Okabe, N., Shimizu, K., Ozaki-Kuroda, K., Nakanishi, H., Morimoto, K., Takeuchi, M. et al. (2004) Contacts between the commissural axons and the floor plate cells are mediated by nectins. Dev. Biol. 273, 244-256.

18) Togashi, H., Miyoshi, J., Honda, T., Sakisaka, T., Takai, Y. and Takeichi, M. (2006) Interneurite affinity is regulated by heterophilic nectin interactions in concert with the cadherin machinery. J. Cell Biol. 174, 141-151.

19) Majima, T., Ogita, H., Yamada, T., Amano, H., Togashi, H., Sakisaka, T. et al. (2009) Involvement of afadin in the formation and remodeling of synapses in the hippocampus. Biochem. Biophys. Res. Commun. 385, 539-544.

20) Hall, A. (1998) Rho GTPases and the actin cytoskeleton. Science 279, 509-514.

21) Zaidel-Bar, R., Cohen, M., Addadi, L. and Geiger, B. (2004) Hierarchical assembly of cell-matrix adhesion complexes. Biochem. Soc. Trans. 32, 416-420.

22) Woodard, A.S., Garcia-Cardena, G., Leong, M., Madri, J.A., Sessa, W.C. and Languino, L.R. (1998) The synergistic activity of $\alpha \mathrm{v} \beta 3$ integrin and PDGF receptor increases cell migration. J. Cell Sci. 111, 469-478.

23) Amano, H., Ikeda, W., Kawano, S., Kajita, M. Tamaru, Y., Inoue, N. et al. (2008) Interaction and localization of Necl-5 and PDGF receptor at the leading edges of moving NIH3T3 cells: implications for directional cell movement. Genes Cells 13, 269284 .

24) Minami, Y., Ikeda, W., Kajita, M., Fujito, T., Amano, H., Tamaru, Y. et al. (2007) Necl-5/ poliovirus receptor cis-interacts with integrin $\alpha_{\mathrm{v}} \beta_{3}$ and enhances its clustering. J. Biol. Chem. 282, 18481-18496.

25) Takahashi, M., Rikitake, Y., Nagamatsu, Y., Hara, T., Ikeda, W., Hirata, K. et al. (2008) Sequential activation of Rap1 and Rac1 small G proteins by PDGF locally at leading edges of NIH3T3 cells. Genes Cells 13, 549-569.

26) Miyata, M., Rikitake, Y., Takahashi, M., Nagamatsu, Y., Yamauchi, Y., Ogita, H. et al. (2009) Regulation by afadin of cyclical activation and inactivation of Rap1, Rac1, and RhoA small G proteins at leading edges of moving NIH3T3 cells. J. Biol. Chem. 284, 24595-24609.

27) Miyata, M., Ogita, H., Komura, H., Nakata, S., Okamoto, R., Ozaki, M. et al. (2009) Localization of nectin-free afadin at the leading edge and its involvement in directional cell movement induced by platelet-derived growth factor. J. Cell Sci. 122, 4319-4329.

28) Abercrombie, M. and Heaysman, J.E. (1953) Observations on the social behaviour of cells in tissue culture. I. Speed of movement of chick heart fibroblasts in relation to their mutual contacts. Exp. Cell Res. 5, 111-131.

29) Fisher, H.W. and Yeh, J. (1967) Contact inhibition in colony formation. Science 155, 581-582.

30) Abercrombie, M. (1979) Contact inhibition and malignancy. Nature 281, 259-262.

31) Thiery, J.P. (2002) Epithelial-mesenchymal transitions in tumour progression. Nat. Rev. Cancer 2, 442-454.

32) Fujito, T., Ikeda, W., Kakunaga, S., Minami, Y., Kajita, M., Sakamoto, Y. et al. (2005) Inhibition of cell movement and proliferation by cell-cell contact-induced interaction of Necl-5 with nectin-3. J. Cell Biol. 171, 165-173.

33) Kakunaga, S., Ikeda, W., Shingai, T., Fujito, T., Yamada, A., Minami, Y. et al. (2004) Enhancement of serum- and platelet-derived growth factorinduced cell proliferation by Necl-5/Tage4/poliovirus receptor/CD155 through the Ras-Raf-MEKERK signaling. J. Biol. Chem. 279, 36419-36425.

34) Sakamoto, Y., Ogita, H., Komura, H. and Takai, Y. (2008) Involvement of Nectin in Inactivation of Integrin $\alpha \mathrm{v} \beta 3$ after the Establishment of Cell-Cell Adhesion. J. Biol. Chem. 283, 496-505.

35) Kajita, M., Ikeda, W., Tamaru, Y. and Takai, Y. (2007) Regulation of platelet-derived growth factor-induced Ras signaling by poliovirus receptor Necl-5 and negative growth regulator Sprouty2. Genes Cells 12, 345-357.

36) Kawano, S., Ikeda, W., Kishimoto, M., Ogita, H. and Takai, Y. (2009) Silencing of ErbB3/ErbB2 signaling by immunoglobulin-like Necl-2. J. Biol. Chem. 284, 23793-23805.

37) Suzuki, K., Hu, D., Bustos, T., Zlotogora, J., Richieri-Costa, A., Helms, J.A. et al. (2000) Mutations of PVRL1, encoding a cell-cell adhesion molecule/herpesvirus receptor, in cleft lip/palateectodermal dysplasia. Nat. Genet. 25, 427-430.

38) Kanzaki, N., Ogita, H., Komura, H., Ozaki, M., Sakamoto, Y., Majima, T. et al. (2008) Involvement of the nectin-afadin complex in PDGFinduced cell survival. J. Cell Sci. 121, 2008-2017.

39) Ooshio, T., Fujita, N., Yamada, A., Sato, T., Kitagawa, Y., Okamoto, R. et al. (2007) Cooperative roles of Par-3 and afadin in the formation of adherens and tight junctions. J. Cell Sci. 120, $2352-2365$.

40) Wakamatsu, K., Ogita, H., Okabe, N., Irie, K., Tanaka-Okamoto, M., Ishizaki, H. et al. (2007) Up-regulation of loricrin expression by cell adhesion molecule nectin-1 through Rap1-ERK signaling in keratinocytes. J. Biol. Chem. 282, 1817318181.

(Received Jan. 26, 2010; accepted Mar. 26, 2010) 


\section{Profile}

Yoshimi Takai was born in 1948 and received his M.D. in 1974 and his Ph.D. in 1980 from Kobe University. He started his research career with studies on protein kinase $\mathrm{C}$ at the late Prof. Nishizuka's laboratory at Kobe University. He was promoted to Assistant Professor in 1975, to Associated Professor in 1981, and to Professor in 1984 at Kobe University. During this research period, he performed pioneering work on the characterization and regulation of small $\mathrm{G}$ proteins. He was moved from Kobe University to Osaka University in 1994. At this university, he identified a novel cell adhesion molecule nectin and this identification provided a new insight into the field of cell adhesion research. For his great achievements on biochemistry and cell biology, he was honored to receive Japanese Biochemistry Society Young Investigator Award in 1982,

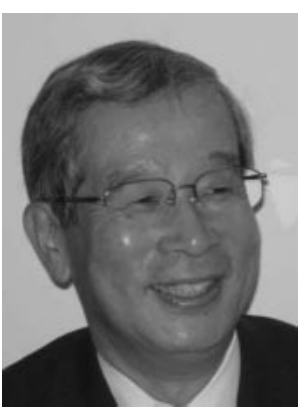

Nissan Science Foundation Award in 1994, Osaka Science \& Technology Award in 1996, Inoue Prize for Science in 1997, and National Prize of Purple Ribbon Medal (Japan) in 2003. He then moved again to Kobe University in 2008 and is the Dean of Graduate School of Medicine at Kobe University. 